\title{
Plan de negocios y plan de marketing en las micro, pequeñas y medianas empresas: análisis bibliométrico
}

\section{Business plan and marketing plan in the MSMEs: bibliometrics analysis}

\author{
VEGA, Tiburcio ${ }^{1}$ \\ GUERRERO, Liliana A. ${ }^{2}$

\section{Resumen} \\ En este artículo se reconoce la importancia de las micro, pequeñas y medianas empresas (MIPYMES) en \\ la generación de empleo y en el número de unidades económicas; es por ello que esta investigación \\ trata de analizar la relación entre los conceptos: plan de marketing, plan de negocios y de alguna manera \\ también la planeación estratégica en las MIPYMES. Se realizó un análisis bibliométrico buscando \\ información en la base de datos Scopus. Se concluye que la producción bibliográfica es limitada y que \\ es poco probable encontrar los conceptos antes mencionados de manera unificada en una misma \\ investigación.
}

Palabras clave: plan de negocios, plan de marketing, MIPYMES

\begin{abstract}
This article recognizes the importance of micro, small and medium-sized enterprises (MSMEs) in generating employment and in the number of economic units; that is why this research tries to analyze the relation between the concepts: marketing plan, business plan and in some way also strategic planning in MSMEs. A bibliometric analysis was performed searching for information in the Scopus database. It is concluded that the bibliographic production is limited and that it is unlikely to find the aforementioned concepts in a unified way in the same investigation.

key words: business plan, marketing plan, MSMEs
\end{abstract}

\section{Introducción}

El interés en la creación de este artículo de investigación, nace de la necesidad de investigar la presencia en plataformas educativas y en buscadores de dos de los grandes temas que impactan en el desarrollo de las MIPYMES: el plan de marketing y el plan de negocios. Estos dos conceptos aparecen en las interacciones cotidianas de las empresas e impactan en la permanencia de las mismas.

Tanto el plan de negocios como el plan de marketing inciden de manera muy cercana y se intersecan en el campo de las MIPYMES, según Zambrano, Navajas y Villamandos (2020), por ello la necesidad de buscar investigaciones realizadas al respecto, de manera unificada.

Aunque al abordarse por primera vez, estos términos parecen ser muy similares, al hacer un análisis más profundo encontramos que tienen fines muy distintos. Un concepto más que se agrega a la discusión es el de planeación estratégica, que aborda elementos relacionados tanto al plan de marketing como al de negocios.

\footnotetext{
${ }^{1}$ Estudiante de doctorado FCA Torreón y maestro de la Facultad de Mercadotecnia, UADEC, tvega@ @otmail.com

${ }^{2}$ Catedrática investigadora FCA Torreón, UADEC, dralilianaclaseaœ@gmail.com
} 
El objetivo de la presente investigación es realizar un estudio bibliométrico en las bases de datos Scopus y ScienceDirect y también apoyar la búsqueda en Google Scholar y en la herramienta multiplataforma Mendeley, con el propósito de esclarecer la interacción de las conceptos: plan de negocios y plan de marketing en las MIPYMES.

A lo largo de este estudio se encontraron investigaciones con títulos como: Plan de negocios para una empresa de consutoría especializada en marketing, finanzas y control de riesgos, sin embargo al examinarla se puede notar que no cuenta con el desarrollo de ambos temas.

Parece ser una generalidad el hecho de que el desarrollo cotidiano de las MIPYMES no tiene un sustento conceptual ni un respaldo académico basado en investigaciones científicas, esto exceptuando trabajos que pudieran existir y que no se hayan encontrado durante el desarrollo del presente artículo. Esto a pesar de que que se buscó información entre las plataformas con mayor recurrencia y en bases de datos de alta calidad científica que están al alcance de los investigadores de las universidades.

El artículo que se presenta está estructurado de la siguiente manera: resumen, introducción, revisión de la literatura, metodología, resultados, conclusiones y referencias bibliográficas.

\subsection{Revisión de la literatura}

Se encontraron coincidencias sobre un aspecto recurrente, tanto en el ámbito académico como en los informes del Instituto Nacional de Estadística y Geografía (INEGI), nos referimos al tema de la planeación como factor fundamental para la permanencia y fortalecimiento de las MIPYMES. Sánchez y Mejia (2017) analizan el hecho de que las empresas le dan mayor importancia a la producción, vista ésta como la generación de bienes y servicios para la venta y obtención de ingresos, que a la planeación.

En la mayoría de los casos las MIPYMES inician sin un plan estratégico, sin un plan de negocios y generalmente también sin un plan de marketing. Lema (2004) indica que si partimos del hecho de que ya se definió el negocio, se debe hacer también un planteamiento estratégico formal donde la misión, la visión, los valores y las metas queden bien establecidas.

El debate acerca de que si son lo mismo la planeación estratégica, el plan de negocios y el plan de marketing, compete absolutamente al desarrollo de esta investigación ya que, si bien estos términos están relacionados y concatenados, son conceptos diferentes con sus propias características muy distintas y claras.

Agila, Vizueta y Tello (2018) afirman que la planeación estratégica, con una perspectiva de lo general a lo particular, es el inicio en el que se debe plasmar la idea del negocio, sus objetivos y sus metas; el plan de negocio será entonces la segunda parte, es decir, se conceptualiza dentro de o como parte de la planeación estratégica.

La planeación estratégica marca el futuro de la empresa, por lo que debe quedar clara la importancia del uso de las tecnologías de la información como parte esencial del negocio para lograr sus objetivos y metas; asimismo al aplicar el marketing, se logrará que el consumidor el centro de esta planeación, así lo indican Patiño y Pinilla (2017).

Una vez definido el conocimiento del rumbo que tomará la empresa, es necesario contar con un documento formal donde se establezca el plan estratégico; también debe definirse a la perfección el plan de negocios y realizar un análisis de FODA (Fortalezas, Oportunidades, Debilidades y Amenazas), éste último servirá para proyetar las ventas e ingresos mediante una estrategia de comercialización analizando el mercado. Ya con toda esta información es posible realizar un documento más completo de análisis económico financiero que brinde la oportunidad de reconocer ingresos y egresos de manera diaria hasta llegar a integrar informes mensuales o anuales que coadyuven a la toma de decisiones. 
Un plan de negocios debería establecer la rentabilidad de la empresa, asi lo expresan Valencia y Paucara (2013). Si bien algunos autores en sus artículos combinan planeación estratégica con plan de negocios, se encargan de esclarecer la diferencia entre el plan de negocios con respecto al plan de marketing.

Debemos comprender que el plan de negocios es muy importante para analizar escenarios futuros y presentes en materia de rentabilidad y factibilidad de la inversión en el negocio, así lo indican Uscategui, Pozo, Espinoza y Beltrán (2018). Los indicadores financieros de rentabilidad como utilidad bruta, utilidad neta, flujo de caja entre otros, deben ser analizados permanentemente por los directivos de la empresa.

Díaz y Pérez (2017) nos dicen que, si bien existen muchos esquemas de planes de negocios, donde incluso en una hoja pueden quedar plasmados, es necesario establecer dentro del mismo los manejos administrativos de ingresos y egresos, así como los de rentabilidad del negocio.

Por otro lado, el plan de marketing es una herramienta dinámica que las MIPYMES deben implementar para mantenerse o crecer. Vidal (2016) establece que un plan de marketing debe tener entre sus apartados principales un análisis de la situación, objetivos, estrategias, plan operativo y presupuesto, así como una evaluación. Durante la aplicación de este plan se desarrollan estrategias como el análisis FODA y el marketing mix.

Tal como se afirma en párrafos anteriores y en coincidencia con Burguete, Romero, Acle y Valverde (2019), si se cuenta con el plan estratégico se tiene definido el rumbo, por lo tanto el plan de marketing será más efectivo porque hay una ruta marcada y las acciones no estarán basadas en caprichos u ocurrencias.

El marketing es útil para satisfacer las necesidades del cliente y para mantener actualizadas las estrategias. Hoy en día existe el marketing dinámico que, por medio de las diferentes herramientas de tecnologías de información y redes sociales, puede ayudar a alcanzar los objetivos y metas de una empresa.

El marketing ofrece también herramientas para la medición de las estrategias digitales, como lo es la publicidad, qué tanto se debe gastar, cuál es el momento indicao para lanzar un promocional, entre otros temas que explica Prasad (2014).

\section{Metodología}

La bibliometría nos ayuda a conocer la importancia y el impacto de las investigaciones en los diferentes ramos y disciplinas. Es una técnica que fundamenta su medición en recursos bilbiográficos y fuentes de información; en el manejo estadístico y cuantitativo de los lectores y de los investigadores que citan los artículos y otros temas, lo que permite dar seguimiento a investigaciones que se han convertido en publicaciones que son consultadas $y$ que a su vez sirven de referencia para otros trabajos académicos.

En esta investigación, se consultaron las bases de datos Scopus y Sciencedirect; si bien Scopus nos ofrece un estudio más completo y exhibe estudios de exigencia en materia de arbitraje e impacto, en su estadística se ve la baja producción científica sobre plan de negocios y plan de marketing en MIPYMES.

Al utilizar Sciencedirect obtenemos mayor cantidad de resultados sobre la búsqueda que nos atañe, sin embargo, esta fuente no permite buscar por citaciones ni usar filtros más avanzados, cosa que sí realiza Scopus. Algo similar ocurre al hacer uso de las demás herramientas como el Mendele, software especializado para búsquedas y gestión de citas y referencias bibliográficas, el cual se usó para consultar artículos que tuvieran referencia a los temas de estudio establecidos y sucedió lo mismo al utilizar Google Schoolar, plataforma en la que se pueden encontrar artículos en español sobre los temas que son de interés en este artículo, pero, como se menciona en este mismo párrafo, ninguna de estas plataformas permite filtros avanzados o búsqueda por citas. 
Por lo anterior, se determina que Scopus será la principal herramienta utilizada para realizar el análisis bibliométrico, sin dejar de lado el respaldo de las demás bases de datos y buscadores ya mencionados.

Una herramienta que también es importante para el desarrollo de esta investigación es la aplicación Google Trends, en la cual se puede obtener una medición del interés que se ha mostrado a lo largo del tiempo en la búsqueda de conceptos usados de manera integrada como marketing plan and business plan en relación a business plan y marketing plan.

Existe una controversia en la manera de citar, debido a la variedad de criterios metodológicos, puesto que, si bien Google Scholar indiza documentos variados: desde investigaciones científicas hasta informes que no tienen una normalización, es por esto que, como mencionan Torres, Ruiz y Delgado (2009) su gran cobertura es complicada.

Se tomó la decisión de realizar la búsqueda en dichos sitios siguiendo la misma metodología de manera que se puedan rescatar los artículos sobre el plan de negocios y el plan de marketing de MIPYMES, privilegiando su importancia por citación, así como por la relevancia que le da cada sitio, cerrando la temporalidad a resultados de los últimos cinco años.

Es importante mencionar que se utilizó el software VOSviewer para construir y visualizar redes bibliométricas.

\subsection{Analizar los conceptos por separado.}

La metodología aplicada en este trabajo está enfocada en exponer la baja producción de artículos de investigación que manejen de manera integral los dos grandes temas sobre las MIPYMES. Es por esto que tanto en las bases de datos como en la demás herramientas utilizadas se realizaron también búsquedas teniendo por separados los conceptos como: plan de negocios, plan de marketing, MIPYMES o MSMEs.

Lo anterior fue necesario por estrategia de investigación, para conocer la diferencia de resultados obtenidos al buscar los tres conceptos juntos y separados.

\section{Resultados}

Al aplicar la búsqueda en Scopus de: business plan and marketing plan nos arroja un resultado de 2718 documentos y, al limitar el filtro a sólo artículos, se reduce a 1605 documentos de una base de datos desde 1963 a la fecha, destacando que en años anteriores, la producción de documentos era baja, para luego notar que en el año 2000 la curva aumenta llegando a su pico mayor en 2006 con 121 artículos, aunque posteriormente inicia un descenso en el año 2013 con 58 artículos y finalmente se aplana la curva al llegar a una modesta producción promedio de 84 artículos a la fecha.

El autor más solicitado en esta base de datos fue Kaplan, R.S., Norton, D.P. con su artículo Having troublewith your strategy? Then map it, escrito en el año 2000 y que es citado 566 veces. 
Tabla 1.

Autores más citados en Marketing y Business Plan

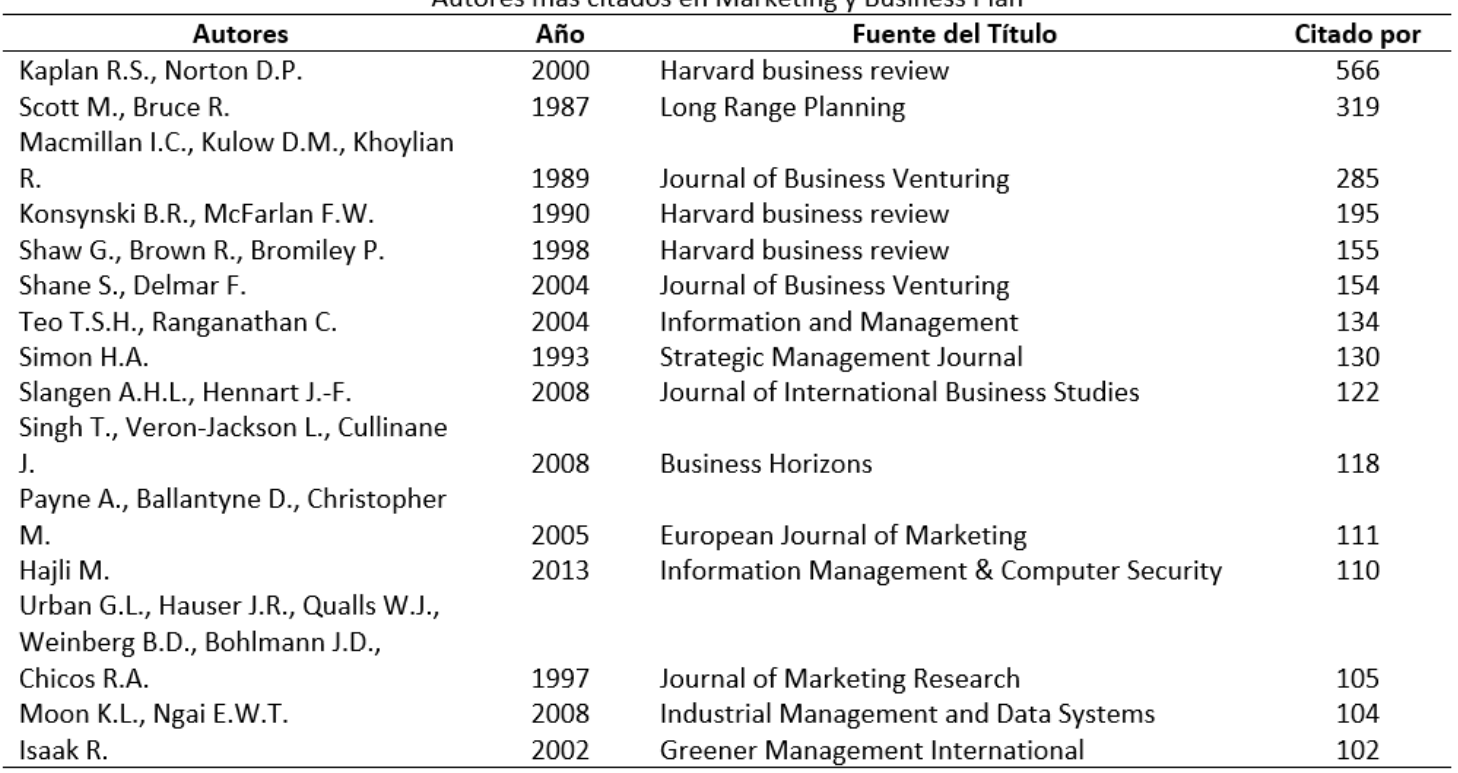

Fuente: Elaboración propia a partir de la base de datos de Scopus, 2020.

Figura 1.

Autores más citados con la búsqueda Business Plan and Marketing Plan

konsynski b.r. (1990ł ban g.l. (1997)

sadrian arnir a. (1994)

payne a. (2005)

simon h.. (1993)

slangen a.b.l. (2008)

moon k.l. (2008)

shaw g.(1998)

hajli m.(2013)

barbieric. (2008) singh 6 (2008)

isaak r. (2002)

wan h.a. (2000)

scott m. (1987)

teo t.s.b. (2004) macmillan i.c. (1989)

kaplan r.s. (2000)

shane S. (2004)

rosenstein j. (1988)

swamidass p.m. (2009)

Fuente: Elaboración propia con datos de Scopus en VOSviewer.

La búsqueda en Scopus usando las palabras clave: business plan and marketing plan a partir de 2015 (para contar información actualizada) obtuvo un resultado de 511 registros, destacando con open access 92 y other con 419 registros, que al aplicar un análisis de resultados arroja los siguientes datos:

El autor que tiene más artículos es Kasemsap K., con 6 artículos, seguido de Cant, M.C., con 4. Por país aparece Estados Unidos en primer lugar con 109 publicaciones y en segundo lugar la India con 52 documentos. Cabe 
mencionar que la búsqueda es a nivel mundial y en idioma inglés, lo que nos confirma una baja producción de documentos.

Tabla 2.

Documentos más citados por autor

\begin{tabular}{|c|c|}
\hline Autor & Documentos \\
\hline Kasemsap, K. & 6 \\
\hline Cant, M.C. & 4 \\
\hline Kosasi, S. & 3 \\
\hline Vedyanto & 3 \\
\hline Abreu, A. & 2 \\
\hline Alén-González, E. & 2 \\
\hline Anbarasi, M. & 2 \\
\hline Baras, J.S. & 2 \\
\hline Charlebois, S. & 2 \\
\hline Chen, YS. & 2 \\
\hline
\end{tabular}

Fuente: Elaboración propia a partir de la base de datos de Scopus, 2020.

Figura 2.

Documentos por autor, desde 2015 a la fecha, mayo 2020

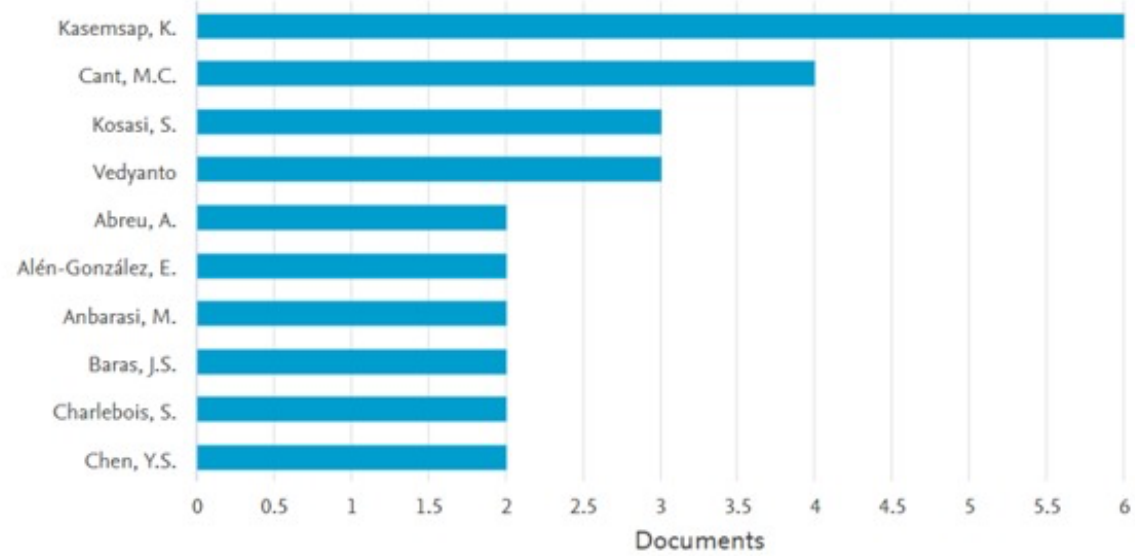

Fuente: Obtenido del análisis de resultados de Scopus.

En lo relacionado a producción de artículo por país, destaca Estados Unidos con 110 documentos. 
Figura 3.

Documentos por país, desde 2015 a la fecha, mayo 2020

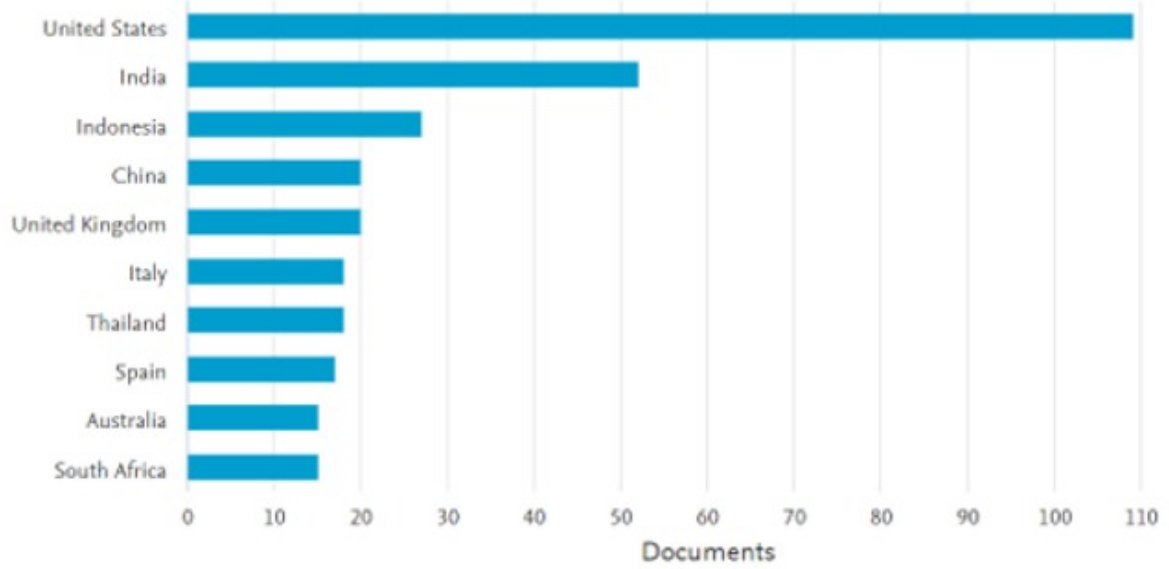

Fuente: Obtenido del análisis de resultados de Scopus.

Con la intención de encontrar en la búsqueda relaciones entre palabras clave, se tomaron las bases de datos de 2015 a la fecha, extraídas de Scopus. Esta decisión arroja una figura muy interesante donde se evidencia que el clúster más importante (indicado con rojo) lo encabeza la palabra Marketing, relacionada cercanamente con los términos: social media, commerce, sales, marketing digital, tourism, entre otras. El clúster, según nos dicen Ovallos, Velez, Figueroa, Sarmiento y Barrera (2017) debe ser visto como una agrupación de vectores de acuerdo con un criterio.

El segundo clúster con color morado lo encabeza la frase: satisfacción al cliente, relacionada con business plan, planeación estratégica, innovación, entre otras, quedando la evidencia de relación entre clústers.

\section{Figura 4.}

Documentos desde el 2015 a la fecha, con búsqueda business plan and marketing plan

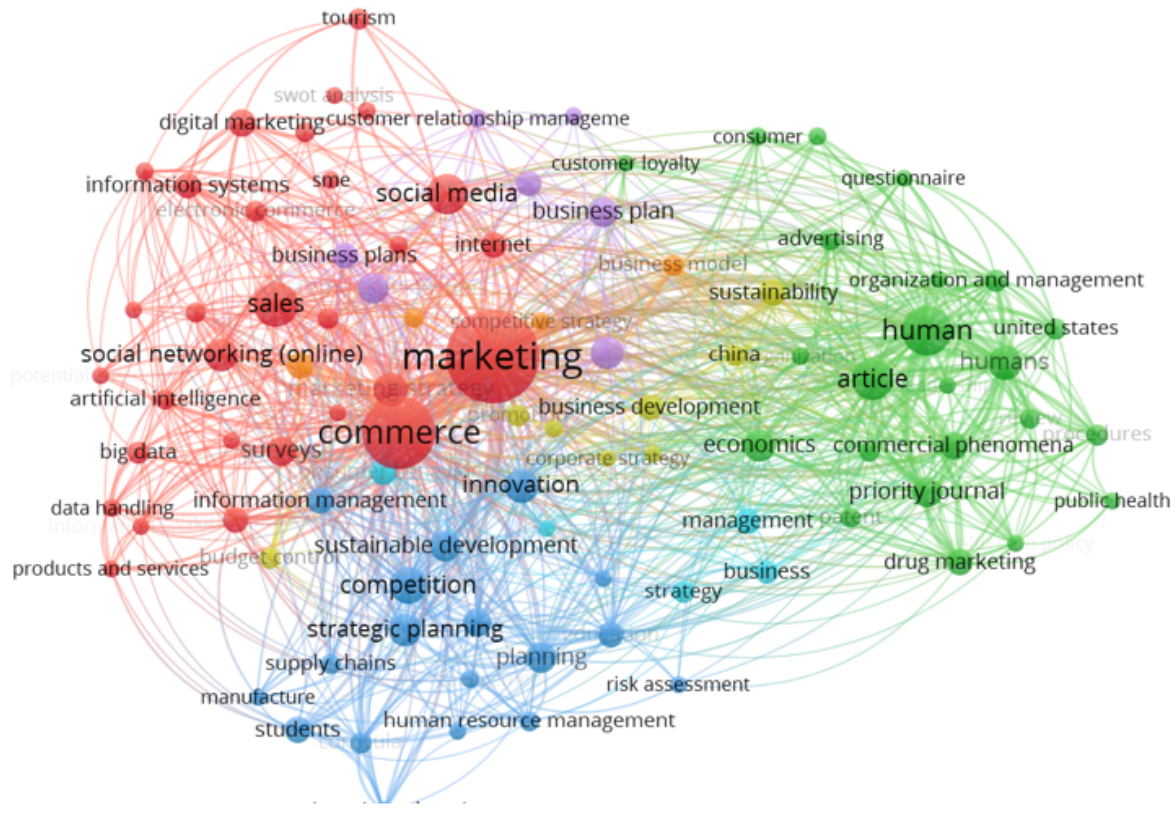

Fuente: Resultados para co-ocurrecia keyword en VosViewer, mayo 2020. 
Si se limita la búsqueda por tipo de documento, el universo se reduce a 320 artículos, por otro lado, al aplicar las siglas MIPYMES en inglés (MSMES) sólo arroja dos documentos: uno publicado en inglés y el otro en español en la Revista Espacios.

De esta base de datos en la que se encontraron 320 artículos, el solicitado es: The roles of system and organizational leadership in system-wide evidence based intervention sustainment: a mixed method study, citado 62 veces y el autor con más producciónes es Cant, M.C., con 4 documentos.

Tabla 3.

Los 5 autores más citados con la búsqueda de artículos con business plan and marketing plan

\begin{tabular}{|c|c|c|c|}
\hline Autor & Titulo & Años & Citado por \\
\hline $\begin{array}{l}\text { Aarons G.A., Green A.E., Trott E., } \\
\text { Willging C.E., Torres E.M., } \\
\text { Ehrhart M.G., Roesch S.C. }\end{array}$ & $\begin{array}{l}\text { The Roles of System and Organizational } \\
\text { Leadership in System-Wide Evidence- } \\
\text { Based Intervention Sustainment: A } \\
\text { Mixed-Method Study }\end{array}$ & 2016 & 62 \\
\hline $\begin{array}{l}\text { Knai C., Petticrew M., Durand } \\
\text { M.A., Eastmure E., James L., } \\
\text { Mehrotra A., Scott C., Mays N. }\end{array}$ & $\begin{array}{l}\text { Has a public-private partnership } \\
\text { resulted in action on healthier diets in } \\
\text { England? An analysis of the Public } \\
\text { Health Responsibility Deal food pledges }\end{array}$ & 2015 & 52 \\
\hline Ley D. & $\begin{array}{l}\text { Global China and the making of } \\
\text { Vancouver's residential property } \\
\text { market }\end{array}$ & 2017 & 31 \\
\hline $\begin{array}{l}\text { Neoh J.G., Chipulu M., Marshall } \\
\text { A. }\end{array}$ & $\begin{array}{l}\text { What encourages people to carpool? } \\
\text { An evaluation of factors with meta- } \\
\text { analysis }\end{array}$ & 2017 & 30 \\
\hline DeCarlo T.E., Lam S.K. & $\begin{array}{l}\text { Identifying effective hunters and } \\
\text { farmers in the salesforce: a } \\
\text { dispositional-situational framework }\end{array}$ & 2016 & 22 \\
\hline
\end{tabular}

Fuente: Elaboración propia con artículos del 2015 a la fecha en Scopus.

Figura 5.

Artículos publicados por autor, del 2015 a la fecha, mayo 2020

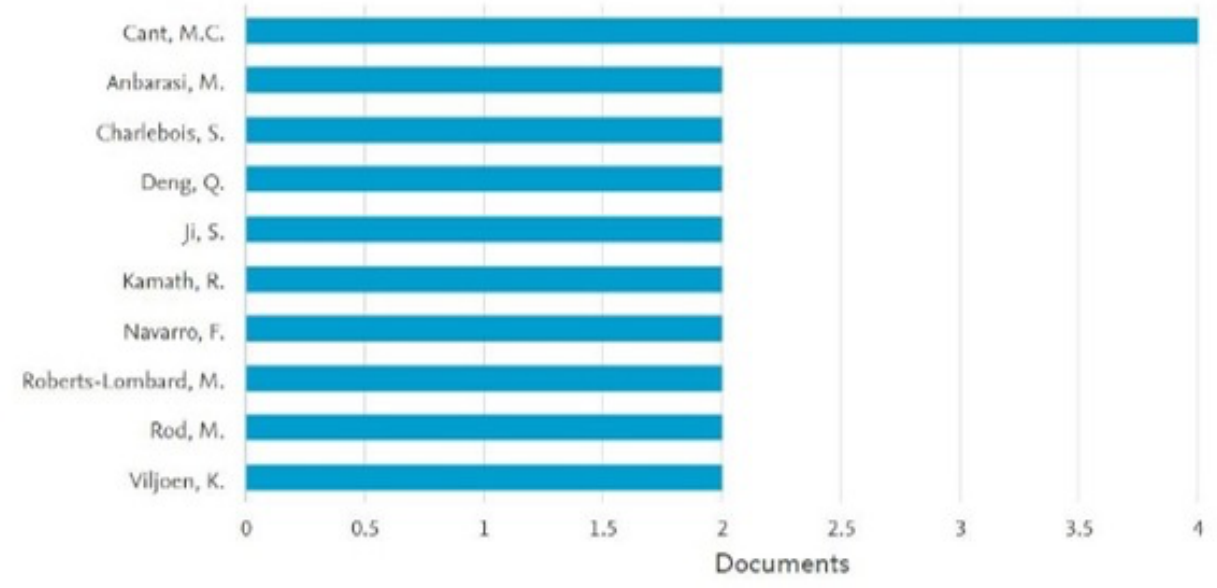

Fuente: Obtenido del análisis de resultados de Scopus.

Como método de comparación se realizó la búsqueda usando el término business plan y se encontraron registros históricos a la fecha con 34,575 documentos, iniciando el despegue de producción de estudios a partir de 1971 
con 14 documentos para llegar a la mayor producción por año en 2014 con 1918 documentos. En 2019 se alcanzaron 1853, manteniéndose la generación de documentos casi constante.

\section{Figura 6.}

Comportamiento histórico de la generación de documentos de business plan
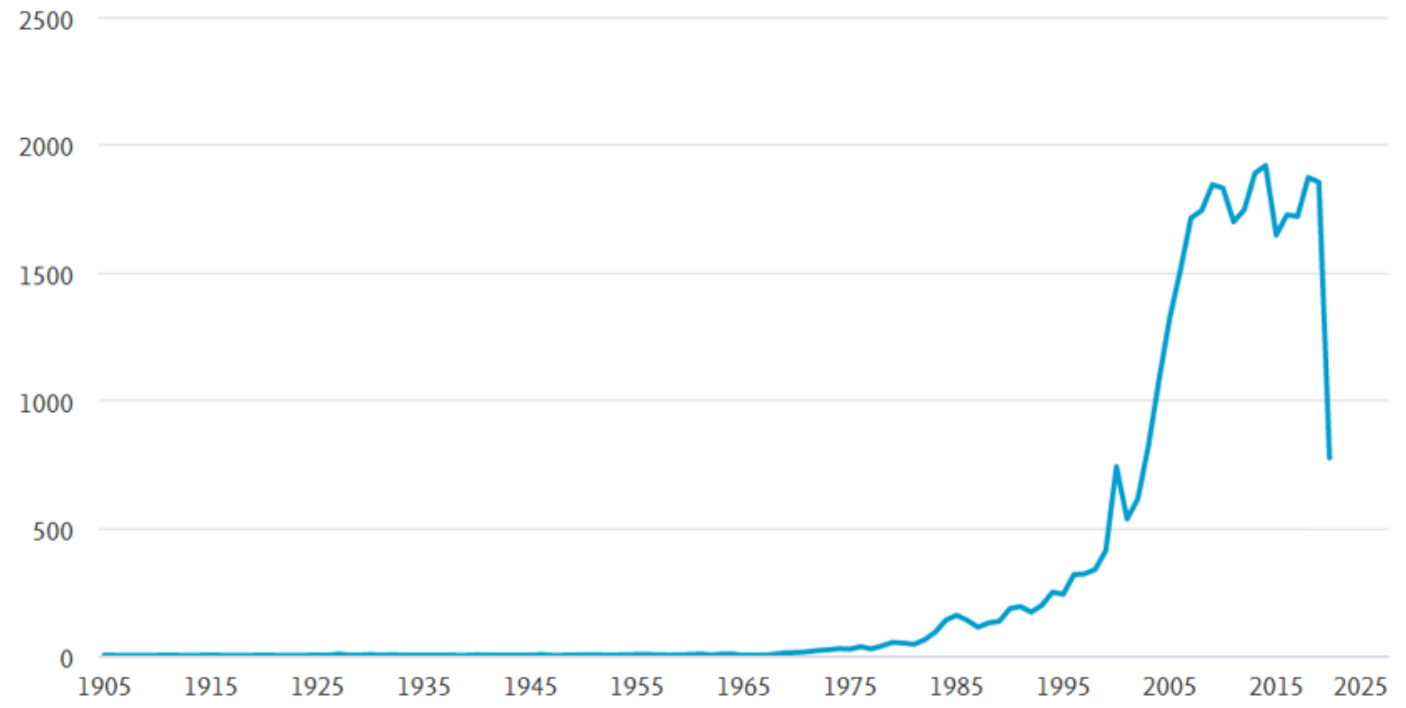

Fuente: Obtenido del análisis de resultados de Scopus, mayo 2020.

Si se analiza por separado en la búsqueda el concepto marketing plan, el resultado lanza 10,689 documentos en la historia de datos a la fecha. A partir de 1971 inicia con 13 documentos mostrando un proceso de aceleración en la generación de estudios sobre el tema, para finalmente alcanzar su pico más alto en 2005 con 753 documentos y en 2019 bajar a 363 documentos.

Figura 7.

Comportamiento histórico de la generación de documentos del marketing plan

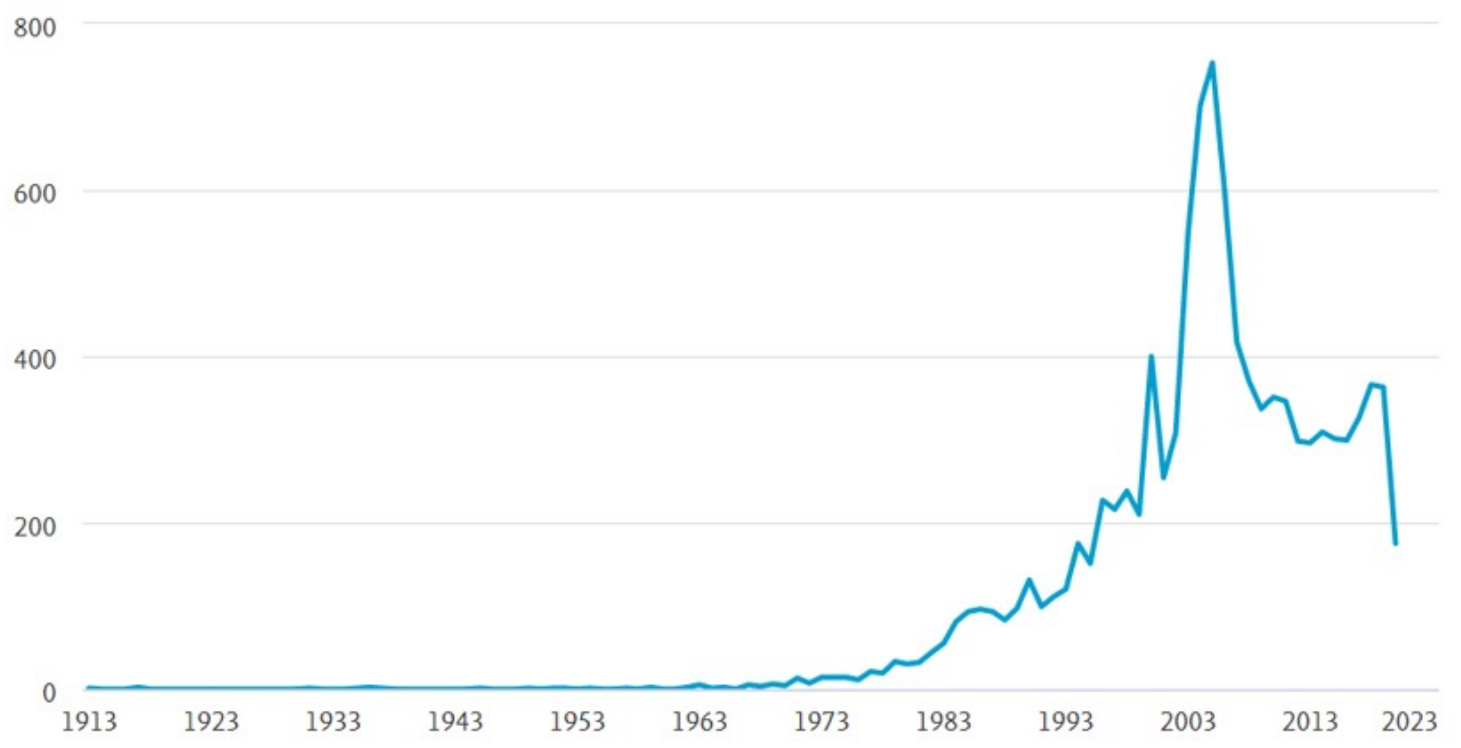

Fuente: Obtenido del análisis del resutados de Scopus, mayo 2020. 
Para el caso de las MIPYMES se realizó la búsqueda y se encontró una baja cantidad de documentos: 590 hasta la fecha, iniciando el despegue a partir de 2011 con 10 documentos y alcanzando el pico más alto en 2019 con 165 documentos, conservando una tendencia a la alta, a pesar de ser pocos los documentos generados.

\section{Figura 8.}

Comportamiento histórico de la generación de documentos de micro, pequeñas y medianas empresas

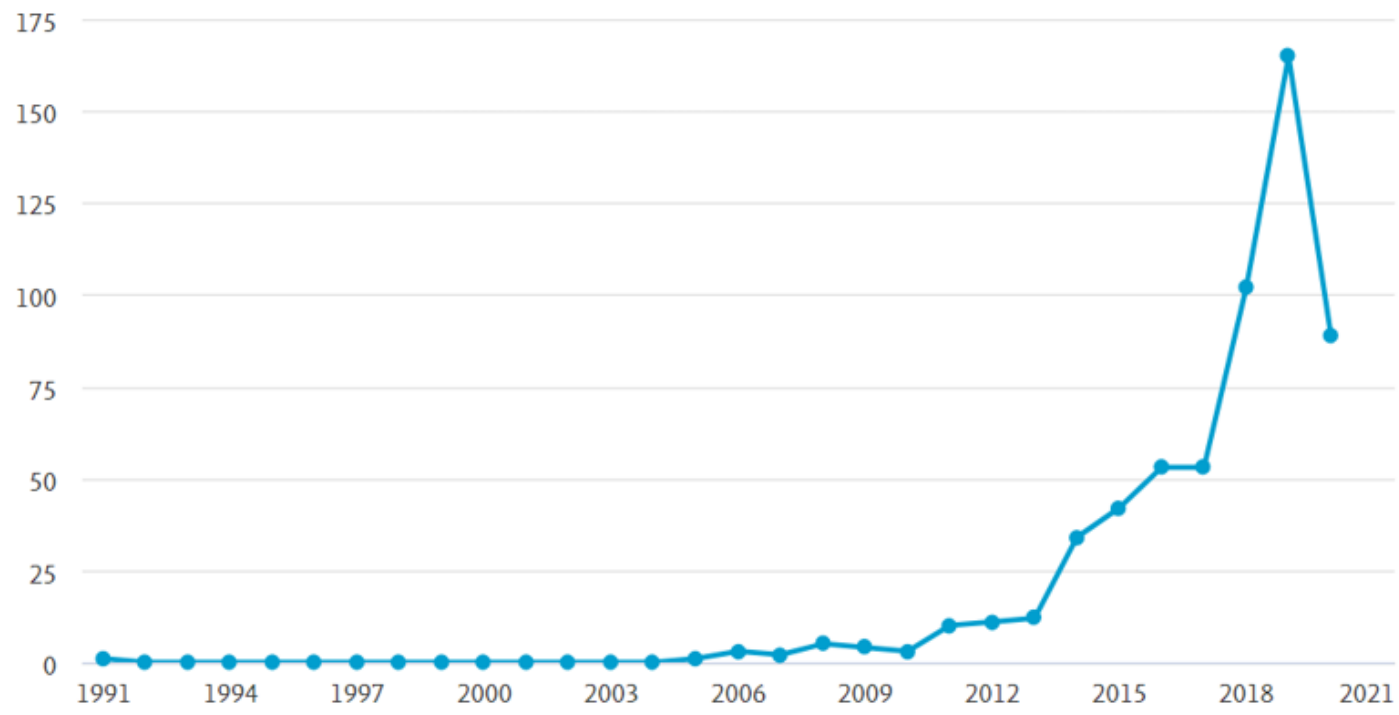

Fuente: Obtenido del análisis de resultados de Scopus, mayo 2020.

\subsection{Datos obtenidos de las otras herramientas}

Al investigar en las demás herramientas tales como Mendeley, Google Scholar y Sciencedirect, fue complicado realizar un análisis bibliométrico debido a que sus bases de datos no están expuestas de manera que permitan realizar un análisis detallado, sin embargo, a su favor tienen la cantidad de artículos sobre el tema de estudio, puesto que al tener más literatura en español se logran adaptar mejor a nuestro caso.

Mediante el uso de Sciencedirect, usando en motor de búsqueda: business plan and marketing plan de 2015 al 7 de junio de 2020, se encontraron 28,608 registros que, al filtrarlos por MIPYMES, (en inglés, MSMEs) nos dio un resultado de 63 registros.

Mientras que, si se aplica la búsqueda usando las palabras: business plan, da como resultado 120,977 registros; usando: marketing plan encontramos 42,268 y con MSMEs 788 resultados.

Lo anterior, evidencia la misma tendencia que en Scopus, es decir, una constante en la baja producción cuando se integran las tres frases clave, aunque con las primeras dos la producción es abundante, nos enfrentamos a una base no comparable por criterios diferentes de elaboración. 


\section{Figura 9.}

Comportamiento de las búsquedas en ScienceDirect, del 2015 a la fecha, mayo 2020

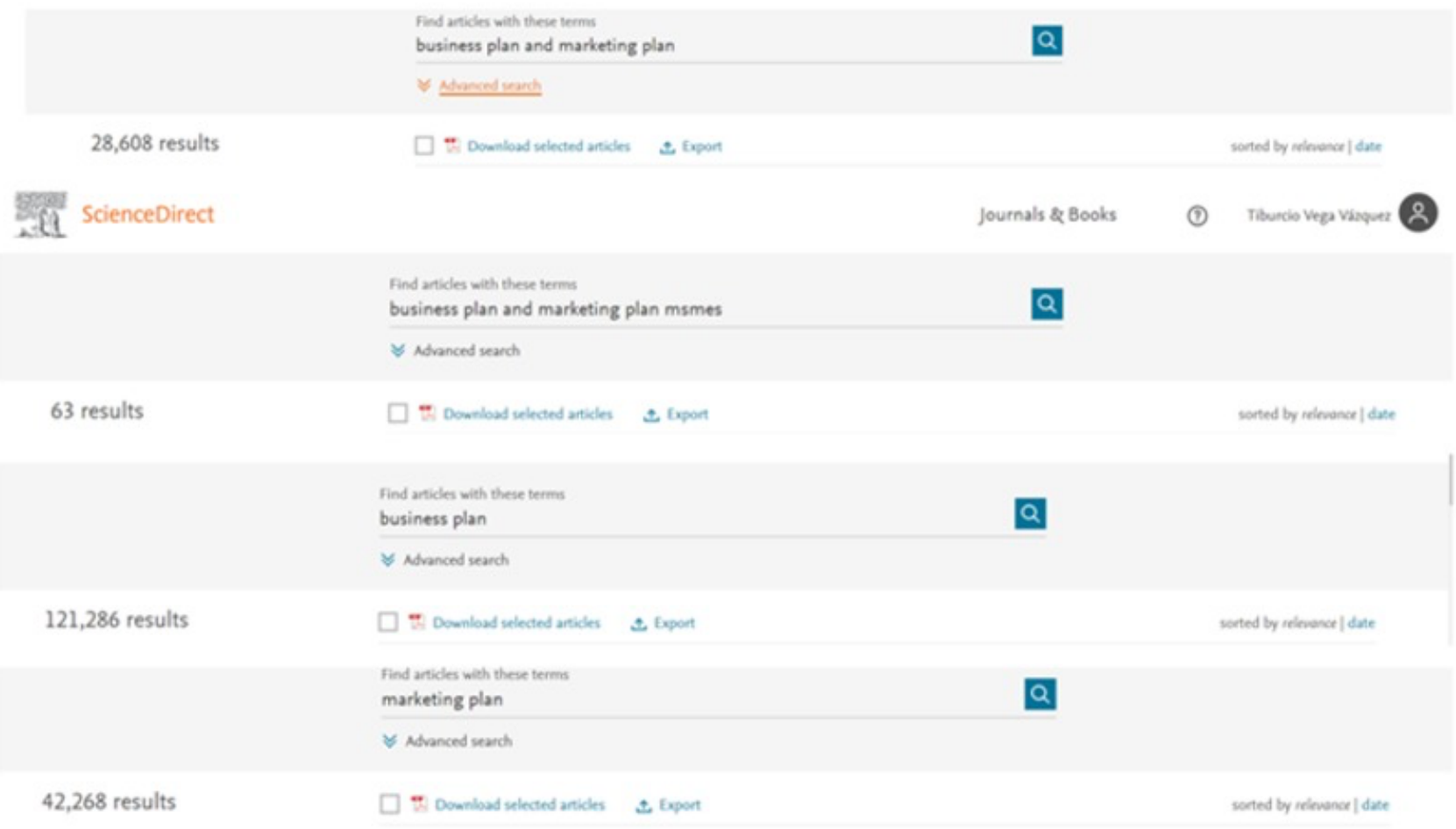

Fuente: Elaboración propia con datos de ScienceDirect.

De los resultados obtenidos de la investigación en Mendeley, sigue la misma tendencia que en Sciencedirect: con la búsqueda mediante business plan and marketing plan MSMEs se obtuvieron 3402 registros, con business plan 38,967 registros y con marketing plan 11,119, prevaleciendo la constante en referencia a que business plan tiene mayor producción que marketing plan.

Si se realiza la búsqueda en español con: plan de negocios y plan de marketing para MIPYMES, nos da un resultado de 1,196 registros, lo cual también es evidencia de que hay más producción en inglés. Lo anterior se refuerza al buscar MIPYMES con 940 registros contra 2,164 de MSMEs. Cabe aclarar que no se pudo filtrar por 2015 a la fecha, sino que se tuvieron que tomar en cuenta todos los años registrados.

Con la aplicación de Google Schoolar se realizó la búsqueda por: business plan and marketing plan MSMEs y se obtuvieron 16,800 registros para el período de estudio de 2015 a la fecha; con business plan 1,020,000 registros y con marketing plan 485,000 registros, siguiendo la misma tendencia en la que que business plan tiene más documentos que marketing plan.

Si se realiza la búsqueda en español con: plan de negocios y plan de marketing para MIPYMES, nos da un resultado de 15,100 registros, conservando la tendencia de mayor producción de artículos en idioma inglés. Sin embargo, en el caso de la búsqueda usando MIPYMES y MSMEs, a diferencia de los resultados anteriores, los registros en español son más que los que están en inglés, dando un resultado de 16,100 y 15,000 respectivamente. 
La plataforma de Google Trends es un importante auxiliar para visualizar estas tendencias, tal como se muestra en las siguientes figuras:

Figura 10.

Ejercicio con Marketing plan and business plan del 2015 a la fecha, mayo 2020

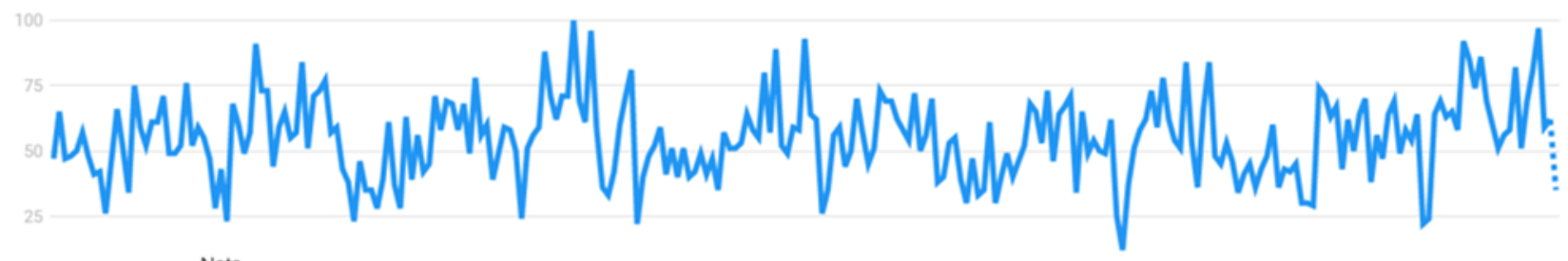

Nota

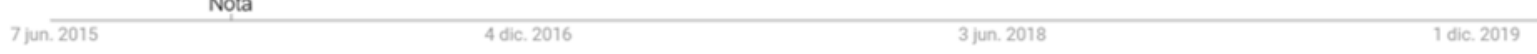

Fuente: Elaboración propia con Google Trends, Interés a lo largo del tiempo.

Figuara 11.

Comparativo de marketing plan and business plan integrado y por separado, interés a lo largo del tiempo
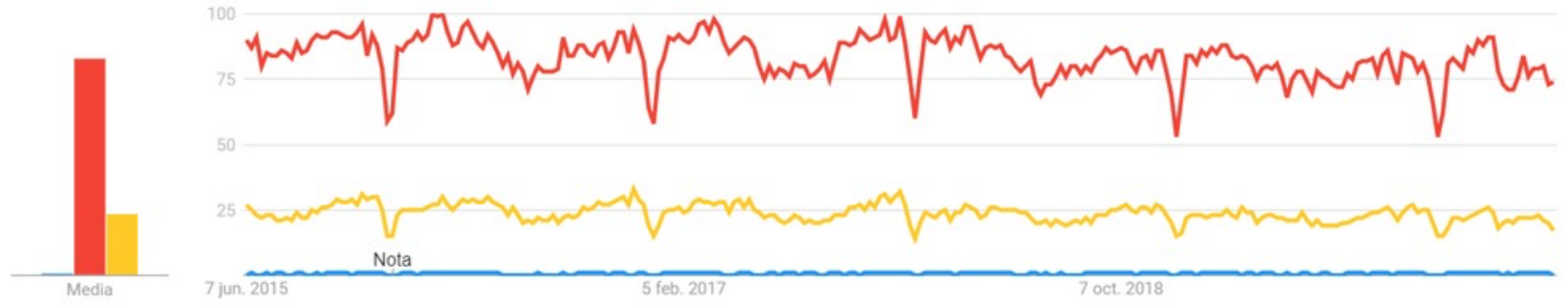

Elaboración propia con Google Trends, mayo 2020. De rojo business plan, de amarillo marketing plan y de azul marketing plan and business plan.

\section{Conclusiones}

Al revisar los resultados obtenidos del análisis de Scopus, se obtienen varias conclusiones, entre las principales está la confirmación de que existe una baja producción científica y de estudios de investigación, que en sus títulos o en el contenido, definan e integren los conceptos: plan de negocios y plan de marketing y si a estos dos términos se le agrega MIPYMES, se reducen aún más los resultados de 302 a 2 . Se observa también una curva de aumento ligera a lo largo del tiempo. La tendencia sigue al revisar los análisis de demás bases de datos, aplicaciones y herramientas de apoyo.

Entre las dificultades que aparecieron en el proceso de investigación, resalta la de homologar y comparar los diferentes artículos por su importancia, ya que cada buscador tiene distintos criterios para definirla.

Se confirma que Scopus, en relación con las otras herramientas utilizadas, es la mejor herramienta de base de datos, esto de acuerdo a su diseño y a la posibilidad de descargar bases de datos de manera sencilla. 
Se concluye también que, la manera de filtrar la información no es homogénea entre los buscadores y que además resulta complicada la búsqueda, puesto que no se permite el análisis de los criterios del índice de citación.

No se encontró, en ninguna de las plataformas (Scopus, Google Scholar, Mendeley y ScienceDirect), un estudio donde se pudiera divisar un análisis de los temas de manera agrupada en una misma investigación, ni tampoco títulos de investigaciones que mezclaran ambos conceptos, es decir plan de marketing y plan de negocios.

Una de las reflexiones que surgieron de esta investigación es el convencimiento de que, si se profundiza en la relación de los términos, pueden surgir productos de investigación de gran calidad que podrán servir de apoyo bibliográfico para quien busque maneras de apoyar y de lograr la permanencia de las MIPYMES.

Es importante destacar que en la búsqueda de bibliografía se encontró un ejemplar en español y otro en inglés de libros que abordan los conceptos de interés en este artículo, editados por el Centro de Estudios Adams durante el año 2018. Parafraseando a Thompson (2013), libros como estos, que se asemejan a manuales o guías generalmente son muy útiles para los emprendedores.

En resumen, del trabajo realizado quedan dos principales hallazgos. El primero es la falta de información sobre los temas de una manera integral y el segundo es la importancia que debe darse a la planeación estratégica como subtema para el plan de marketing y el plan de negocios.

Para futuras investigaciones consideramos conveniente lograr el acceso total a Scopus y a las demás herramientas tecnológicas, para acceder de manera total a los artículos que sean causa de análisis en trabajos posteriores.

\section{Referencias bibliográficas}

Agila, M. V., Vizueta, S. E., \& Tello, G. E. (2018). La elaboración de un plan de negocios como alternativa para el desarrollo empresarial. Revista Espacios, 39(50), 3-5. Obtenido de https://www.revistaespacios.com/a18v39n50/a18v39n50p01.pdf

Andia, W., \& Paucara, E. (2013). Los planes de negocio y los proyectos de inversión: similitudes y diferencias. Industria data, 16(1), 80-81. Obtenido de Redalyc.org

Burguete, M. A., Romero, E. D., Acle, R. S., \& Valverde, M. L. (2019). El impulso de la planeación estratégica en el proceso de marketing de la industria manufacturera de Puebla México. Revista Internacional de administración y finanzas, 12(1), 3-17. Obtenido de https://papers.ssrn.com/sol3/papers.cfm?abstract_id=3462723

Centro de Estudios ADAMS. (2018). Marketing y plan de negocios de la microempresa. Bogotá: Ediciones de la U.

Díaz, J., Pérez, P. (2017) Universidad Técnológica del Perú. Obtenido de http://repositorio.utp.edu.pe/handle/UTP/2577

Lema, J. (2004). La guía estratégica el corazón del plan estratégico. EIA(2), 10-11. Obtenido de http://www.scielo.org.co/pdf/eia/n2/n2a02.pdf

Ovallos, D., Velez, J., Figueroa, A., Sarmiento, J., \& Barrera, J. (2017). Conocimiento y desarrollo socioeconómico, una revisión de la literatura. Revista Espacios, 38(46), 14. Recuperado el junio de 2020, de http://www.revistaespacios.com 
Prasad A.(2014). Marketing dynamics: a primer on estmation and control. Foundations and trends in marketing, 9(3), 175-177. doi:10.1561/1700000031

Patiño, E., \& Pinilla, S. (2017). Investigación y anáisis como fundamento para la planeación estratégica del mercadeo digital. Revista Espacios, 38(41), 2,3. Obtenido de http://www.revistaespacios.com/

Sánchez, J., Vázquez, G., \& Mejía, J. (2017). La mercadotecnia y los elementos que influyen en la competitividad de las MIPYMES comerciales en Guadalajara, México. NNOVAR, 27(65), 95-96. Obtenido de https://doi.org/10.15446/innovar.v27n65.65064.

Thompson M. (2013). The small business start-up guide. Naperville, Illinois, USA: Sourcebooks.

Torres, D., Ruiz R., \& Delgado E. DANIEL, T.,(2009). Google scholar como herramienta para la evaluación científica. El profesional de la información, 18(5), 501-505. doi:DOI: 10.3145/epi.2009.sep.03

Uscategui, C., Pozo, B., Espinoza, M. \& Beltrán, A. (2018) Principales métodos de evaluación de inversión para futuros emprendedores en Ecuador. Revista Espacios, 39(24), 3-6. Obtenido de https://www.revistaespacios.com/a18v39n24/18392423.html

Vidal, P. (2016). Metodología para la elaboración de un plan de marketing online. 3 ciencias, 5(2), 57-72. doi:https://doi.org/10.17993/3cemp.2016.050226.57-72

Zambrano-Santos, Z., Navajas-Romero, V., \& Ceular-Villamandos, N. (2020). Los emprendimientos y perspectivas de financiación: análisis bibliométrico. Revista Espacios, 41(3), 1-2. Obtenido de http://www.revistaespacios.com/a20v41n03/a20v41n03p27.pdf

Esta obra está bajo una Licencia Creative Commons Attribución-NoCommercial 4.0 International

(cc) BY-NC 